\title{
Analysis of passive calorimetric probe measurements at high energy influxes
}

\author{
Thorben Kewitz $^{1 *} \mathbb{D}$, Maik Fröhlich ${ }^{2}$ and Holger Kersten ${ }^{1}$
}

\author{
* Correspondence: \\ kewitz@physik.uni-kiel.de \\ ${ }^{1}$ Institute of Experimental and \\ Applied Physics, Kiel University, Kiel \\ 24098, Germany \\ Full list of author information is \\ available at the end of the article
}

\begin{abstract}
High energy influxes increase the complexity of passive calorimetric probe measurements because of an increasing temperature of the surrounding materials like the probe holder. This leads to a distinctively different evolution of the probe temperature compared to low energy influxes. Different established methods for the analysis of passive calorimetric probe data are presented and it is shown that they are not applicable for high energy influx measurements resulting in the requirement of a novel analysis approach. Such an approach is given in this paper by the combination of the exponential and the linear method. The high energy influx measurements are studied for a commercial atmospheric pressure plasma jet, in order to illustrate our suggested modified approach.
\end{abstract}

Keywords: Calorimetric probe, High energy influx, Atmospheric pressure plasma jet, Plasma diagnostics

\section{Introduction}

Atmospheric pressure plasma jets are used for cleaning, activation and thin film coating of metal, plastics and other materials $[1,2]$. But often they have a large energy flux onto surfaces because of input powers in the $\mathrm{kW}$ range. As the thermal stress of a substrate is a key parameter in many different plasma applications, in particular, for temperature-sensitive materials, detailed knowledge is needed for the optimization of processes involving these sources. The thermal conditions not only define process limitations, but also influence the quality of coatings [3]. Yet, atmospheric plasma sources with a high energy influx in the order of $100 \mathrm{~W} / \mathrm{cm}^{2}$ towards substrate surfaces offer some advantages compared to low power sources $\left(<1 \mathrm{~W} / \mathrm{cm}^{2}\right)$, e.g., faster treatments of substrates and effective use of precursors.

Calorimetric probes are a well-known and widespread diagnostic tool for the determination of the energy influx $[4,5]$. They can be divided into active and passive calorimetric probes. The active probes are heated to a constant temperature and the heating power is recorded. Any additional energy influx from a plasma source results directly in a reduced heating power. However, only energy influxes smaller than the heating power can be measured, which puts a limit to this diagnostic. The limit of the probe used so far are a few watts per square centimeter and can, therefore, not be used for high energy influxes [6]. The passive probes can be distinguished for different principles, again. For example, the probes are exposed to the energy source on the one side

(c) The Author(s); licensee Springer on behalf of EPJ. 2017 Open Access This article is distributed under the terms of the Creative Commons Attribution 4.0 International License (http://creativecommons.org/licenses/by/4.0/), which permits unrestricted use, distribution, and reproduction in any medium, provided you give appropriate credit to the original author(s) and the source, provide a link to the Creative Commons license, and indicate if changes were made. 
of the probe and cooled on the other side until a stationary temperature distribution is reached [7]. This leads to a spatial temperature gradient along the probe from which the energy influx can be derived. The other principle uses smaller probes, which are considered to have a negligible temperature gradient in the heated part of the probe during the plasma interaction. These probes based on temporal changes of the temperature have been used in many different designs for versatile processes, mostly at low pressure and small energy flux applications. A suitable review can be found in [5].

It was already shown that for the investigation of atmospheric pressure plasma jets with a large power consumption changes in the design of the probe are necessary [8]. These adaptions allow to handle the high mechanical and thermal stress of the probe induced by the investigated high power atmospheric pressure plasma jet from the company Plasmatreat.

In this present study we will focus on the analysis of the results obtained with modified passive probes. First, we explain the well-known linear and exponential methods $[4,9-11]$ and the underlying assumptions in detail, before we show for a typical measurement why these methods are not suitable for energy influxes in the order of $100 \mathrm{~W} /$ $\mathrm{cm}^{2}$. Finally, we introduce a novel method by combining the linear and the exponential methods and show that this approach is suitable for the analysis of high energy influxes by a passive calorimetric probe.

\section{Basics of the analytical method at low energy fluxes}

The basis for all the common methods for the analysis of passive calorimetric probe measurements is the consideration of temporal temperature changes during the heating and the cooling phase of the calorimetric probe [9]:

$$
C_{p} \frac{\mathrm{d} T_{p}}{\mathrm{~d} t}=P_{\text {in }}-P_{\text {out }}
$$

with the heat capacity of the probe $C_{p}$, the time derivative of the probe temperature $T_{p} . P_{i n}$ is the net incoming power containing the heating processes as well as losses due to plasma-wall-interactions. A list of the various contributions to the thermal energy balance at the substrate (probe) can be found in $[12,13] . P_{\text {out }}$, on the other hand, includes all loss processes dependent on the rising temperature of the probe. The processes are heat radiation (Stefan-Boltzmann law)

$$
P_{\text {rad }}=\varepsilon \sigma A_{p}\left(T_{p}^{4}-T_{e q}^{4}\right)
$$

with the emissivity $\varepsilon$, the Stefan-Boltzmann constant $\sigma$, the area of the probe $A_{p}$ and the equilibrium temperature $T_{e q}$, and thermal conduction

$$
P_{\text {cond }}=c\left(T_{p}-T_{e q}\right),
$$

with a constant $c$ depending on the material and geometry of the probe.

It includes, furthermore, the virtual reduction of the incoming power due to higher substrate temperatures. This reduction is often considered to be a loss in order to distinguish this effect from any other changes of the energy influx $[4,10]$. As soon as the substrate temperature rises, the contributions of the total energy flux to the substrate which are dependent on the substrate temperature decline, generally. For example, under atmospheric pressure one of these contributions is that of the heated neutral gas. 
The energy transferred from the gas to the substrate will diminish with a rising substrate temperature.

At low pressure free convection can be neglected. When using atmospheric pressure plasma jets, forced convection, which can be treated linearly analog to thermal conduction, may become dominant during the plasma treatment.

The three loss processes, radiation, conduction, and convection, are also those which are present in the cooling phase after plasma treatment. Here, the convection losses are dependent on the experimental conditions. If the gas flow is switched off together with the plasma or if the plasma jet is moved away from the probe, free convection is present. If the gas flow is still on, the losses caused by forced convection depend on the temperature of the gas.

The incoming power can be determined by a comparison of the change of temperature during the heating $\left(P_{\text {in }}-P_{\text {out }}\right.$, plasma/energy influx on, eq. 4.1$)$ and the cooling phase ( $\left(P^{\prime}{ }_{\text {out }}\right.$, plasma/energy influx off, eq. 4.2$)$ leading to eq. 4.3:

$$
\begin{aligned}
& C_{p}\left(\frac{\mathrm{d} T_{p}}{\mathrm{~d} t}\right)_{\text {heat }}=P_{\text {in }}-P_{\text {out }} \\
& C_{p}\left(\frac{\mathrm{d} T_{p}}{\mathrm{~d} t}\right)_{\text {cool }}=-P_{\text {out }}^{\prime} \\
& P_{\text {in }}=C_{p}\left(\left(\frac{\mathrm{d} T_{p}}{\mathrm{~d} t}\right)_{\text {heat }}-\left(\frac{\mathrm{d} T_{p}}{\mathrm{~d} t}\right)_{\text {cool }}\right)+\left(P_{\text {out }}-P_{\text {out }}^{\prime}\right)
\end{aligned}
$$

It is important to point out that the power loss functions during the two phases $P_{\text {out }}$ $\left(T_{p}\right)$ and $P^{\prime}{ }_{\text {out }}\left(T_{p}\right)$ are not necessarily the same. A crucial point for the losses to remain the same is the reduction of the incoming power due to higher substrate temperatures. In the case of a plasma jet this loss is mainly caused by the temperature difference between the probe and the neutral gas. As soon as the probe temperature rises, the difference becomes smaller and the incoming power is reduced. To mirror this effect during the cooling phase, a gas flow equally large to that one during the heating phase and at equilibrium temperature has to be applied. However, this is often not achievable due to residual heat in the jet or other effects on the gas temperature. Turning the gas flow off leads to free convection and, thereby, again to different power loss contributions.

Another issue is the temperature of the surrounding, i.e., the probe holder. The power losses in eqs. 2 and 3 are dependent on this temperature, which is the equilibrium temperature $T_{e q}$ at the beginning of the treatment. If the probe holder heats up during the measurement these losses are no longer solely dependent on the probe temperature $T_{p}$. Hence, a time dependent component is introduced and, therefore, the power loss functions $P_{\text {out }}\left(T_{p}\right)$ and $P_{\text {out }}^{\prime}\left(T_{p}\right)$ differ from each another.

To illustrate these relations and the consequences for the measurement of high energy influxes, we start with the methods used for the analysis of small energy influxes and discuss their applicability to high energy influxes.

The simplest approach to apply the relation given in eq. 4.3 is the linear method. At the beginning of the heating curve at the equilibrium temperature $T_{e q}$ the losses are negligible and the energy influx is proportional to the slope of the temperature curve leading to [9]: 


$$
P_{\text {in }}=\left.C_{p}\left(\frac{\mathrm{d} T_{p}}{\mathrm{~d} t}\right)_{\text {heat }}\right|_{T=T_{\text {eq }}}
$$

Since this method is applied at the beginning of the heating curve, the incoming power should be fully applied in a short time and the recording frequency of the probe temperature should be sufficiently high to minimize uncertainties for the determination of the slope $\left(\frac{\mathrm{d} T_{p}}{\mathrm{~d} t}\right)_{\text {heat }}$. For higher energy influxes the time, where the probe temperature is close to the equilibrium temperature, becomes very short and it is not always possible to apply the full power to the probe in the time available. This may be attributed to a starting phase of the source plasma or moving it over the probe if it was started elsewhere to account for the starting phase. There will always be a short time where the probe is exposed only to a fraction of the total energy flux of the source and where it is already heated up.

Another method is the exponential analysis. With the assumptions of a constant incoming power, linear loss terms, and a constant temperature for the surrounding of the probe the shape of the signal for heating and cooling phases will be exponential $[9,10]$ :

$$
\begin{aligned}
& T_{p, \text { heat }}(t)=\left(T_{\text {eq }}+\frac{P_{\text {in }}}{a}\right)-\left(\frac{P_{\text {in }}}{a}\right) \exp \left(-\frac{a}{C_{p}} t\right) \\
& T_{p, \text { cool }}(t)=T_{e q}+\left(T_{p, s t}-T_{e q}\right) \exp \left(-\frac{a}{C_{p}} t\right)
\end{aligned}
$$

Here $a$ is the constant for the linear cooling processes and $T_{p, s t}$ the temperature of the probe at the beginning of the cooling phase.

Because of the constant temperature of the surroundings, i.e., $T_{e q}$, the power loss functions $P_{\text {out }}\left(T_{p}\right)$ and $P^{\prime}{ }_{\text {out }}\left(T_{p}\right)$ are equal and cancel each other out (eq. 4.3) leading to [4]:

$$
P_{\text {in }}=C_{p}\left(\left(\frac{\mathrm{d} T_{p}}{\mathrm{~d} t}\right)_{\text {heat }}-\left(\frac{\mathrm{d} T_{p}}{\mathrm{~d} t}\right)_{\text {cool }}\right)
$$

In this case, the incoming power can be simply calculated with the difference of the time derivatives of the temperature $\dot{T}_{p}=\frac{\mathrm{d} T_{p}}{\mathrm{~d} t}$ during the heating and the cooling phase (Fig. 1a). Fig. 1b shows this relation for an exemplary measurement in a low pressure calibration experiment with an electron beam. It can be seen, that the curves of $\dot{T}_{p}$ for the heating and cooling phase are linear and parallel, so that their difference is roughly constant.

The assumptions made in this case are valid for short measurements with small energy influxes where the temperature of the surrounding materials, i.e., the probe holder, does not change significantly and for probe temperatures where heat loss by radiation is negligible.

If the probe is used for measurements at higher temperatures, i. e. heated up by sources with high energy influx, thermal radiation becomes relevant and the energy losses can no longer be assumed to be linear (see eq. 2). But, if the energy losses are solely dependent on the probe temperature (constant $T_{e q}$ ), the function of the power losses $P_{\text {out }}$ represented by the cooling curve $\dot{T}_{p}$ is still the same for each energy influx measurement. Therefore, eq. 7 can be applied [12]. 

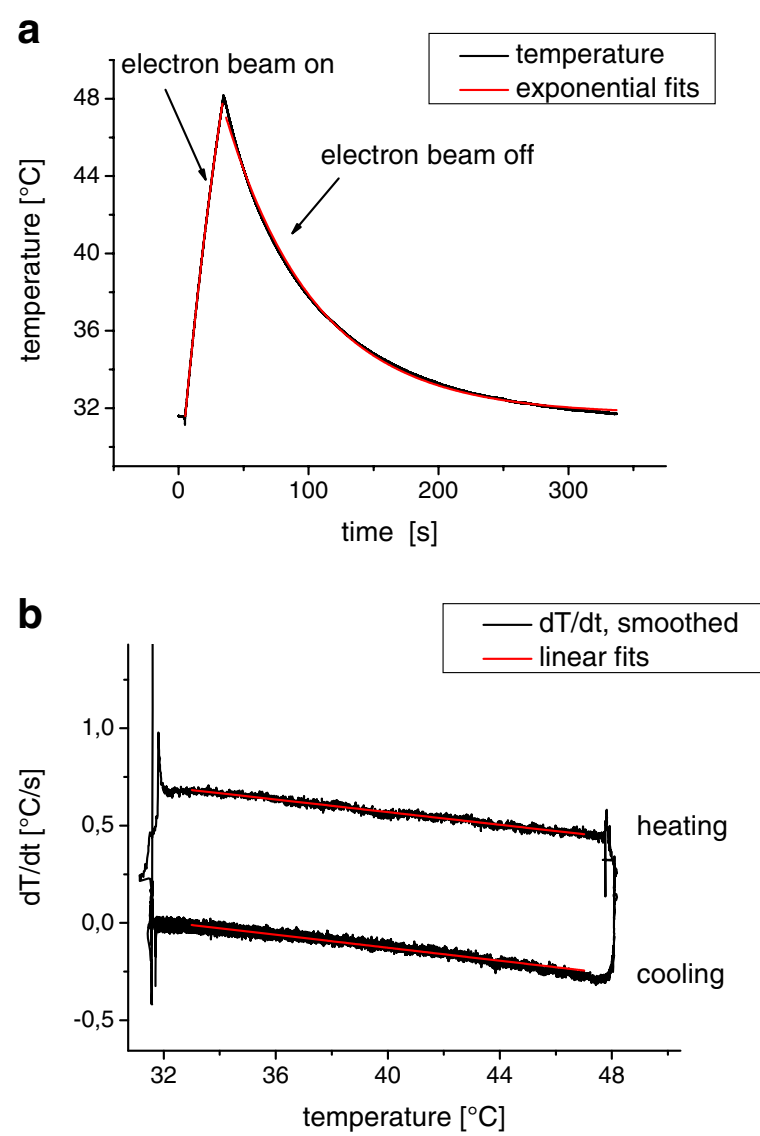

Fig. 1 Measurement for a small energy influx of $0,043 \mathrm{~W} / \mathrm{cm}^{2}$ in a calibration experiment at low pressure $\left(6.6 \cdot 10^{-5} \mathrm{~Pa}\right)$ for a passive calorimetric probe. Electrons emitted from a heated tungsten wire $\left(j=15 \mathrm{kA} / \mathrm{cm}^{2}\right)$ are accelerated to the biased probe $\left(V_{\text {bias }}=950 \mathrm{~V}\right)$ during the heating phase (for further information see [16]). Graph a shows the temperature evolution over time. $\mathbf{b}$ shows the relation between the temporal derivation of the temperature and the temperature for the same measurement

However, if plasma sources with a high energy influx onto substrates are used, the boundary conditions change. The assumption inherent to the cases above that the power losses are only dependent on the probe temperature can only be made if the surroundings of the probe, i.e., the probe holder, are at equilibrium temperature $T_{e q}$. But due to the high energies the temperature of the holder may increase remarkably during the treatment. Hence, the temperature $T_{e q}$ in eqs. 2 and 3 is time dependent and, thereby, the power loss function $P_{\text {out }}$. This means that the power loss functions $P_{\text {out }}$ $\left(T_{p}\right)$ and $P^{\prime}{ }_{\text {out }}\left(T_{p}\right)$ in eqs. 4.1 and 4.2 are not the same. As a consequence, eq. 7 can no longer be used for the whole measurement and the method has to be modified.

\section{Modified analytical method at high energy fluxes and experimental verification for plasma jet operation}

The concerns raised in the discussion of the linear and exponential methods concerning high energy influx measurements are illustrated by a typical measurement for high energy influx in Fig. 2. This measurement was performed at the atmospheric pressure plasma jet system from the company Plasmatreat [14] consisting of a FG5001 generator, a HTR12 transformer and a RD1004 nozzle (similar to [15]). A metal sheet 

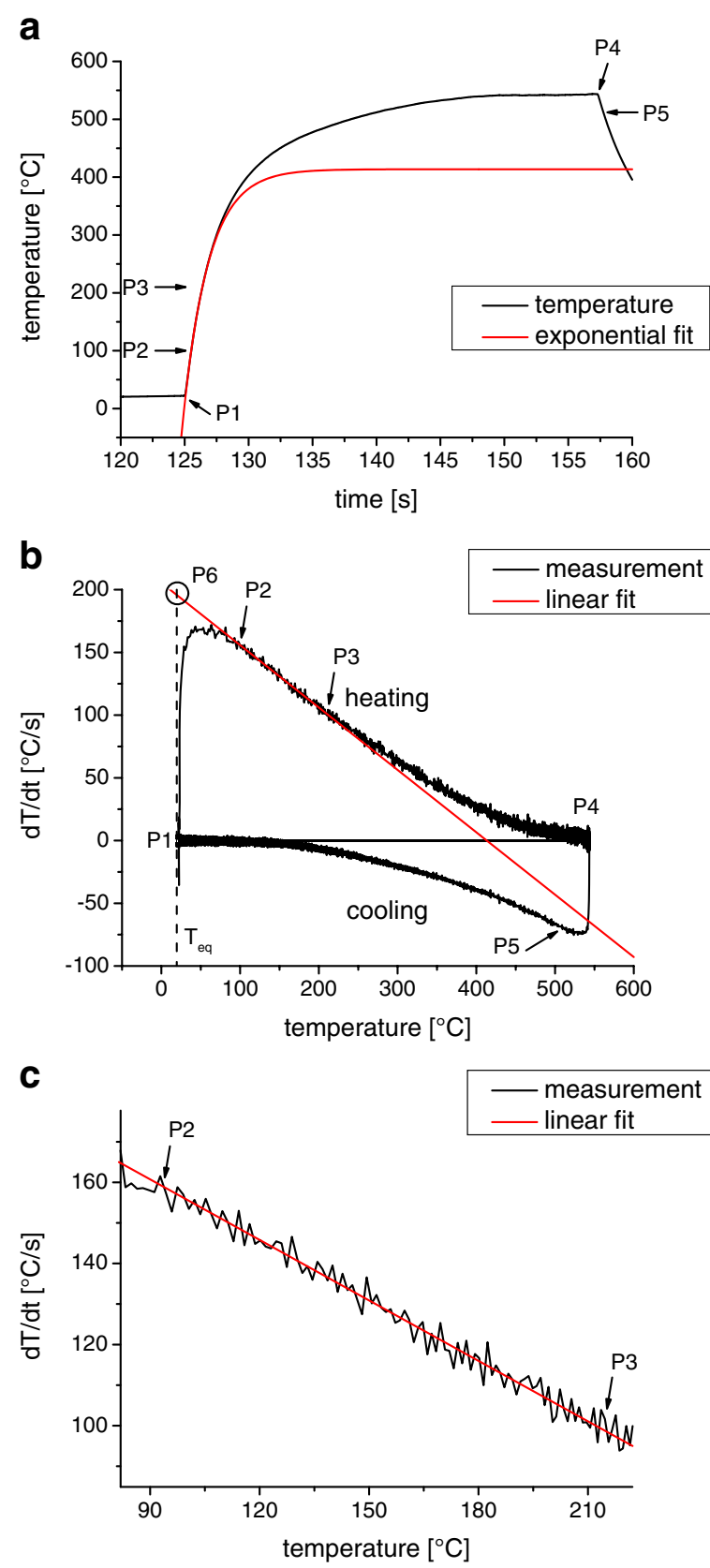

Fig. 2 Calorimetric probe measurement for the energy influx of an atmospheric pressure plasma jet. The jet was operated with a voltage of $300 \mathrm{~V}$ (primary power $2.3 \mathrm{~kW}$ ), a frequency of $19 \mathrm{kHz}, 30 \mathrm{slm}$ nitrogen and at a distance to the probe of $6 \mathrm{~mm}$. The measurement frequency was $100 \mathrm{~Hz}$ and the energy influx was determined to $433 \pm 2 \mathrm{~W} / \mathrm{cm}^{2}$. a shows the temperature evolution over time with an exponential fit. The exponential fit corresponds to the linear fit between P2 and P3 of b. $\mathbf{b}$ shows the relation between the temporal derivation of the temperature and the temperature for the same measurement. Different phases can be distinguished. P1 marks the start of the heating, where the shield for the warm-up time was removed from between the plasma jet and the probe. P2 is the point where the full power is applied to the probe. The surrounding starts to heat up around P3 and the measured curve deviates from the fits (linear and exponential). The plasma jet was turned off at P4 and the transition to the cooling phase was completed around P5. The dashed line marks the equilibrium temperature and the intersection between this line and the linear fit (P6) gives the value for the linear method to obtain the energy influx. c shows the part for the linear fit in more detail. It can be seen that the measurement frequency is sufficiently high and the linear fit is in good agreement with the data 
positioned in between the plasma jet and the probe is used to shield the probe during the warm-up time of the jet (see Fig. 3). Once stable conditions are reached the metal sheet is removed and the plasma jet interacts with the probe (P1). As the gas flow does not adjust immediately to the new geometry, the incoming power rises between P1 and P2 until, again, stable conditions are reached and the incoming power is fully applied at P2. From this point on until the point marked with P3 the loss terms are linear, resulting in a linear decrease of $\dot{T}_{p}$ over $T_{p}$, indicating that the surrounding is still at $T_{e q}$ and thermal radiation can be neglected (P2 and P3 are chosen as the limits of this linear decrease). At P3 the temperature of the surrounding starts to increase significantly and the curve deviates from the linear fit (red line) to higher values. This process surpasses also the increasing thermal radiation which should let the measured curve deviate to smaller values compared to the linear fit. P4 marks the time where the plasma jet is switched off and the beginning of the transition phase to the cooling curve which starts at P5.

The two methods described so far depend on a constant temperature $T_{e q}$ of the surrounding. This temperature can be considered as constant between P1 and approximately P3 and also between P4 and P5, because of the short time between these two points.

Due to the changing temperature of the surrounding after P3 the curves do not have an exponential shape and the exponential method cannot be applied. The same applies to the curve before P3, because there is no corresponding part in the cooling curve with the same temperature of the surrounding.

The beginning of the heating phase was already considered for the linear method. The consequences of the concerns mentioned for the use of this method for high energy influxes can be seen clearly in Fig. $2 \mathrm{~b}$. The probe temperature is already around $100{ }^{\circ} \mathrm{C}$ when the full power is applied to the probe (P2) and the losses cannot be neglected.

The second area, where the temperature of the surrounding can be assumed as constant, the transition between the heating and the cooling phase (P4-P5) is also complex if plasma jets are used. The difficulties to get the same power losses were discussed above. The consequences can again be recognized in Fig. 2b. The decrease after P4 is much smaller than the increase after P1 due to residual heat in the gas flow and can, thus, not be used for the measurement of the energy influx. In conclusion, both, the

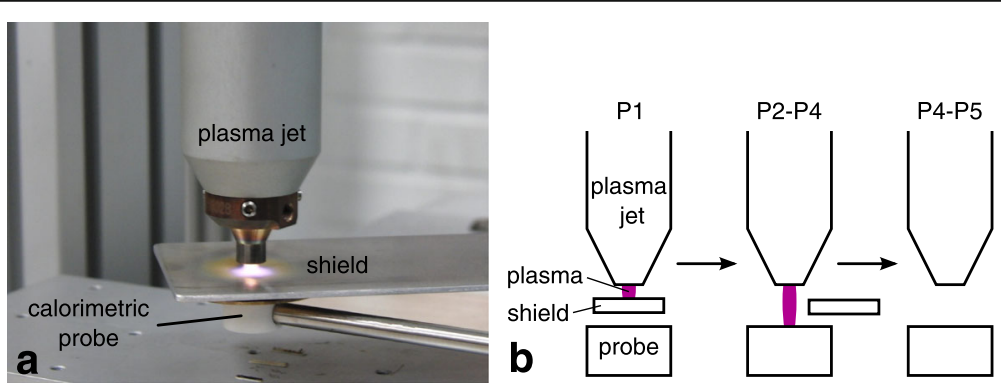

Fig. 3 Photograph $\mathbf{a}$ and schematic illustration $\mathbf{b}$ of the experimental setup and process. The shield separates plasma and probe until its removal at P1 
linear and the exponential method cannot be used for the analysis of the energy influx in this case.

So, we have shown that the established methods lead to remarkable errors in the determination of the energy influx in this case. Therefore, a modified method is presented in the following.

To overcome the difficulties mentioned, we decided to take the part of the heating curve where $P_{\text {out }}\left(T_{p}\right)$ is linear (see Fig. $2 \mathrm{~b}$ and c between P2 and P3 and compare to the exponential method) and extrapolate the linear fit to the equilibrium temperature (see Fig. $2 \mathrm{~b}$ at P6). This procedure results in the slope of the temperature curve which we would get at equilibrium temperature if the full power would be applied to the probe immediately and this value is exactly what is used for the linear method. Therefore, we apply the linear method (eq. 5) to obtain the energy influx of $433 \pm 2 \mathrm{~W} / \mathrm{cm}^{2}$ in the given experiment. An additional uncertainty of about $10 \%$ is induced by the calibration of the probe. Using the Gaussian profile of the jet obtained at the same parameters with air (standard deviations of $3.35 \pm 0.06 \mathrm{~mm}$ and $3.47 \pm 0.06 \mathrm{~mm}$ in $\mathrm{x}$ - and $\mathrm{y}$ directions [8]) and regarding the probe diameter of $5 \mathrm{~mm}$, this results in a total energy flux from the jet of $1.61 \pm 0.16 \mathrm{~kW}$. This value is comprehensible considering the primary power of $2.3 \mathrm{~kW}$ used for this experiment, since a lower value was expected due to different losses in the plasma jet system and only a fraction of the total energy is transferred to the surface. A closer look at the part for the linear fit is presented in Fig. 2c. It illustrates in more detail that the linear fit is in good agreement with the data and that the amount of measured points is sufficient for this method.

\section{Conclusion}

The passive calorimetric probe measurements at high energy influxes show distinctively different temperature evolutions compared to measurements at low energy influxes. The slopes of the heating and cooling curves differ substantially from each other due to the changing temperature of the surrounding material. The analysis methods used so far in the literature are based either on a constant temperature $T_{e q}$ of this surrounding or, with the linear method, a fast application of the full power to the measuring probe. Since neither of these conditions is fulfilled by the investigated atmospheric pressure plasma jet a combination of the exponential and the linear method was used. This combination omits the necessity of a fast application of the full power by using the exponential part of the heating curve and in the same time does not require the surrounding to be close to the equilibrium temperature for a long time. The successful application for the investigation of energy fluxes in the order of $100 \mathrm{~W} / \mathrm{cm}^{2}$ was presented.

\section{Acknowledgements}

The authors would like to thank the company Plasmatreat for supplying the plasma jet system. The authors would also like to thank V. Rohwer, M. Poser, and T. Riedel for the technical assistance and F. Haase for the calibration measurement (Fig. 1a and b). This work was supported by the German Federal Ministry of Education and Research (BMBF) under Project FKZ: 13 N12220. 
Author details

${ }^{1}$ Institute of Experimental and Applied Physics, Kiel University, Kiel 24098, Germany. ${ }^{2}$ Leibniz Institute for Plasma

Science and Technology, Greifswald 17489, Germany.

Received: 11 July 2016 Accepted: 19 December 2016

Published online: 19 January 2017

\section{References}

1. Penkov OV, Khadem M, Lim WS, Kim DE (2015) A review of recent applications of atmospheric pressure plasma jets for materials processing. J Coat Technol Res 12(2):225-235

2. Fröhlich M, Bornholdt S, Regula C, Ihde J, Kersten H (2014) Determination of the energy flux of a commercial atmospheric-pressure plasma jet for different process gases and distances between nozzle outlet and substrate surface. Contrib Plasma Phys 54, No. 2:155-161

3. Thornton JA (1974) Influence of apparatus geometry and deposition conditions on the structure and topography of thick sputtered coatings. J Vac Sci Technol 11, no. 4:666-670

4. Thornton JA (1978) Substrate heating in cylindrical magnetron sputtering sources. Thin Solid Films 54:23-31

5. Bornholdt S, Fröhlich M, Kersten H. Calorimetric Probes for Energy Flux Measurements in Process Plasmas. In: Bonitz M, Becker K, Lopez J, Thomsen H, editors. Complex Plasmas, Springer Series on Atomic, Optical, and Plasma Physics, 82. Switzerland: Springer; 2014. ISBN 978-3-319-05437-7.

6. Wiese R, Kersten H, Wiese G, Bartsch R (2015) Energy influx measurements with an active thermal probe in plasma-technological processes. EPJ Tech Instrum 2:2

7. Steffen $\mathrm{H}$, Kersten $\mathrm{H}$, Wulff $\mathrm{H}$ (1994) Investigation of the energy transfer to the substrate during titanium deposition in a hollow cathode arc. J Vac Sci Technol A 12(5):2780-2783

8. Kewitz T, Fröhlich M, von Frieling J, Kersten H (2015) Investigation of a Commercial Atmospheric Pressure Plasma Jet by a Newly Designed Calorimetric Probe. IEEE Trans Plasma Sci 43, no. 5:1769-1773

9. Hussla I, Enke K, Grünwald H, Lorenz G, Stoll H (1987) In situ silicon-wafer temperature measurements during RF argon-ion plasma etching via fluoroptic thermometry. J Phys D Appl Phys 20:889-896

10. Kersten H, Kroesen GMW (1990) The thermal balance of substrates during plasma processing. Contrib Plasma Phys 30(6):725-731

11. Bornholdt S, Kersten H (2013) Transient calorimetric diagnostics for plasma processing. Eur Phys J D 67:176

12. Kersten H, Deutsch H, Steffen H, Kroesen GMW, Hippler R (2001) The energy balance at substrate surfaces during plasma processing. Vacuum 63:385-431

13. Bornholdt S, Ye J, Ulrich S, Kersten H (2012) Energy fluxes in a radio-frequency magnetron discharge for the deposition of superhard cubic boron nitride coatings. J Appl Phys 112:123301

14. Plasmatreat. www.plasmatreat.de. Accessed 08 Feb 2016

15. Regula C, Lukasczyk T, Ihde J, Fladung T, Wilken R (2012) Corrosion protection of metal surfaces by atmospheric pressure plasma jet treatment. Prog Org Coat 74:734-738

16. Stahl M, Trottenberg T, Kersten H (2010) A calorimetric probe for plasma diagnostics. Rev Sci Instrum 81:023504

\section{Submit your manuscript to a SpringerOpen ${ }^{\circ}$ journal and benefit from:}

- Convenient online submission

- Rigorous peer review

- Immediate publication on acceptance

- Open access: articles freely available online

- High visibility within the field

- Retaining the copyright to your article 